\title{
Cathodic pretreatment improves the resistance of boron-doped diamond electrodes to dopamine fouling
}

Raphaël Trouillon, ${ }^{*}$ Yasuaki Einaga, ${ }^{b, c}$ and Martin A. M. Gijs ${ }^{a}$

a Laboratory of Microsystems, Ecole Polytechnique Fédérale de Lausanne, CH-1015 Lausanne, Switzerland. Email address: raphael.trouillon@m4x.org

b Department of Chemistry, Faculty of Science and Technology, Keio University, 3-14-1 Hiyoshi, Yokohama 223-8522, Japan.

c JST-CREST, 3-14-1 Hiyoshi, Yokohama 223-8522, Japan

The resistance of cathodically and anodically treated boron-doped diamond electrodes to dopamine fouling was investigated. It was found, using cyclic voltammetry and electrochemical impedance spectroscopy, that the cathodic preparation offers an increased resistance to fouling, in addition to an enhanced electrochemical response.

\section{Introduction}

Boron-doped diamond (BDD) has attracted increasing attention as a reliable and robust electrode material. This surface has shown its potential in water treatment and ozone generation, and its increased resistance to dopamine (DA) or serotonin foulings [1-3] makes it a promising candidate for bio-analysis [4-7].

Even though the native BDD is hydrogen-terminated, its surface can be modified by adequate pretreatments. Cathodic or anodic pretreatments in acids have been found to regenerate a fouled BDD electrode [8] and to alter the response of BDD, the cathodic one leading to enhanced electrochemical responses [9]. Different preparation procedures for BDD have been found to show different electrochemical responses and to allow for the separation of DA and ascorbic acid oxidation signals [5]. In this communication, the effect of pretreatment on DA fouling has been investigated.

\section{Experimental}

Ferrocenemethanol (FcMeOH), hexaammineruthenium (III) chloride (RuHex), DA, and sulphuric acid (98\%) were all obtained from Sigma-Aldrich. Phosphate buffered saline (PBS, $\mathrm{pH}=7.4$ ) was obtained from PAA Laboratories GmbH. Polydimethylsiloxane (PDMS) was obtained from Dow Corning, USA. Deionised water was used throughout the experiments. As described elsewhere [1], the boron doped polycrystalline diamond films were grown on p-type (111) silicon wafers (Furuya Metal 
Co., Japan) using microwave plasma enhanced chemical vapour deposition. Boron-to-carbon (B/C) ratio in the gas phase was here fixed at $0.1 \%$.

Before the electrochemical tests, the BDD surface was ultrasonicated in isopropyl alcohol for 5 minutes, rinsed successively in isopropyl alcohol and water, and then dried with compressed air. The BDD electrode was delimited by clamping a PDMS gasket ( $\sim 1 \mathrm{~mm}$ thick) featuring a $\varnothing 5 \mathrm{~mm}$ hole onto the BDD surface, thus defining a recessed electrode. The electrode was then introduced into a custom-made cell, allowing for electrochemical analysis in few $\mathrm{mL}$ of liquid. Eye observation revealed that no leakage between the PDMS and the BDD surface occurred during the experiments. This system was kept assembled for the duration of the pretreatment and electrochemical tests. The setup was completed with an $\mathrm{Ag} \mid \mathrm{AgCl}, 3 \mathrm{M} \mathrm{KCl}$, reference electrode (Bioanalytical Systems Inc., USA) and a Pt wire as a counter electrode. All the experiments were carried out with an Ivium Powerstat (Ivium, Netherlands) functioning in the 3-electrode mode and run in aerated buffers.

The electrodes were pretreated for 5 minutes in $0.5 \mathrm{M} \mathrm{H}_{2} \mathrm{SO}_{4}$. The potentials were $-3 \mathrm{~V}$ and $3 \mathrm{~V}$ for the cathodic and anodic pretreatments, respectively. This preparation procedure was carried out before each set of experiments. The charge $Q_{p}$ transferred during the pretreatments is shown in Table 1. After the pretreatment, the electrode was rinsed twice with water, and was immediately used. The electrode was not left to dry or significantly exposed to the atmosphere after the pretreatment or between the experiments.

The cyclic voltammograms (CV) were performed in PBS (1 mM FcMeOH, $1 \mathrm{mM}$ RuHex or $1 \mathrm{mM} \mathrm{DA})$ at $100 \mathrm{mV} \mathrm{s}^{-1}$. The oxidative peak current $i_{p}$, measured as the current difference between the oxidation peak and the extrapolated baseline, and the peak separation $\Delta E_{p}$, the difference of potential between the two redox peaks, were both obtained from each trace. The electrochemical impedance spectroscopy (EIS) scans were performed in $1 \mathrm{mM}$ DA in PBS. The starting potential was $0.3 \mathrm{~V}$, the amplitude $20 \mathrm{mV}$. The frequency range was $1 \mathrm{MHz}-1 \mathrm{~Hz}$. The EIS data was analysed using the built-in facility of the Ivium software.

X-ray photoelectron spectroscopy (XPS) analyses were performed on the cathodically and anodically-prepared surfaces with an Axis Ultra spectrometer (Kratos Analytical, Manchester, UK) using an Al Ka source.

\section{Results and Discussion}

The XPS data is presented in Figure 1A. After the cathodic treatment, only a small O1s peak (in comparison to the C1s peak) can be observed. The relative intensity of this oxygen peak increases after the anodic procedure, thus indicating an increased oxygen content of the surface after this pretreatment.

Initially cyclic voltammograms (CVs) for DA-unfouled electrodes were performed at cathodically and anodically prepared electrodes, for FcMeOH [10] and DA (Figure 1B, C). The FcMeOH CVs for the anodic treatment show a slightly decreased current in comparison to the cathodic one, even though no significant differences are observed from the CV numerical characteristics ( $\mathrm{p}=0.04$ for $i_{p}$ and $\mathrm{p}=0.1$ for $\Delta E_{p}$; Table 1 ). To ensure this observation was not due to variation in the 
electrode geometry, CVs were carried out in $1 \mathrm{mM}$ RuHex. The results obtained for the two pretreatments were not significantly different, as expected from an outer-sphere redox probe. This establishes that the system geometry is stable during the pretreatments. The fact that the CVs for RuHex, for the two pretreatments, are similar also ensures there are no noticeable semi-conducting behaviour for this electrode.[11] The slightly more sluggish reaction of FcMeOH on the anodically-treated surface could therefore indicate a limited surface-dependence, as reported for instance for ferro/ferricyanide.[12] The electrode capacitance was obtained from i-V curves run in PBS. No significant differences were observed (cathodic: $1.4 \pm 0.3 \mu \mathrm{F} \mathrm{cm}{ }^{-2}$; anodic: $1.8 \pm 0.5 \mu \mathrm{F} \mathrm{cm}{ }^{-2} ; p=0.41 ; n=3$ ).

For DA oxidation (right part of the curves of Figure 1C), the anodic treatment, produces a more oxygenated surface, induces a significant loss, as determined from a Student's t-test, of a third of the peak current, as well as a significant widening of the peak separation. Altogether, these observations indicate a facilitated DA oxidation reaction at the cathodically treated electrode in agreement with previous reports [9].

After the CV characterization, DA fouling was induced by polarizing the electrode at $0.8 \mathrm{~V}$ for 15 minutes in 1 mM DA in aerated PBS, for the two different electrode pretreatments. This concentration is much higher than expected in vivo in the brain, or in vitro in cultured cells, but allows for a rapid and reproducible model for DA fouling. The electrode was placed vertically during the fouling procedure, to avoid interferences due to gravity, probably induced by the sedimentation of non-adsorbed insulating films [2,3]. The traces were largely comparable for the two pretreatments (Figure 1D), as indicated by the similar charge $Q_{f}$ transferred through the electrode during the fouling procedure $(\sim 6.5 \pm 1.1 \mathrm{mC})$, even though the cathodic preparation induces a slightly higher current. A slight shoulder (indicated by the arrow on Figure 1D) can also be observed for the cathodically-treated surface, an indication of the growth of the DA insulating sites on the electrode [13].

Figure 2A-D shows CVs performed before and after the fouling procedure, for the different experimental conditions. Fouling generally induces a decrease in the measured current. This decrease is significant for FcMeOH, for the two treatments, but is more important after the anodic treatment, and the shape of the CV is dramatically altered. For the case of DA oxidation, the electrode appears to be completely fouled after anodic treatment, as no oxidative peak can now be observed over the range of potentials considered. However, for the cathodically-treated surface, a well-defined CV can still be observed, despite a serious drop in current and a higher peak separation. Altogether, the CV data show that the extent of fouling is more severe on the oxygenated surface. As $Q_{f}$ is similar for the two types of electrodes, the same amount of fouling products should have been produced, and similar levels of fouling could be expected. This fact strongly suggests that the insulating material produced during DA oxidation has a better affinity for the anodically-treated BDD. Similarly, EIS was used to investigate the state of the electrode before and after fouling (Figure 2E, F). The resulting data was analysed using Randles' model, and two parameters of interest are reported in Table 1: the solution resistance $R_{S}$ and the charge transfer resistance $R_{c t}[14]$. The double layer capacitance $C_{d l}$ was also extracted from the data, its value 
was in good agreement with the ones obtained from $\mathrm{i}-\mathrm{V}$ curves at unfouled electrodes $\left(\sim 1.3 \pm 0.6 \mu \mathrm{F} \mathrm{cm}^{-2}\right)$ and did not significantly change with pretreatment or fouling. The diffusion limited regime was not properly observed over the considered range of frequencies, and the Warburg impedance was therefore not considered. Before fouling, and in comparison to the cathodic treatment, the anodic preparation induced a higher $R_{c t}$, hinting a facilitated reaction. This is in good agreement with the higher current observed for DA oxidation. However, the higher currents obtained from the CV at the cathodically-pretreated surface cannot be solely explained by higher heterogenous electron transfers. Other factors are also expected to contribute to this observation [11]. The anodic pretreatment led to a significantly higher $R_{s}$. This observed increase in resistance may arise from the increased oxygen content observed from the XPS spectra, leading to partial insulation of the electrode [9] or maybe to a limited decrease in surface conductivity due to the presence of oxygen, as it has been reported for non-doped diamond samples [15]. After fouling, for both electrodes, a large increase in $R_{c t}$ indicates a decrease in electrochemical activity. This is particularly relevant for the surface-sensitive DA oxidation. However, the $R_{c t}$ obtained after fouling is significantly larger on the anodically treated surface than for the cathodic pretreatment, indicating more sluggish electron transfer. Interestingly, this is in good agreement with the CV data, where no well-defined CV for DA could be recorded after fouling on the anodically-treated BDD, thus further demonstrating the poorer resistance to fouling of this surface.

From a molecular point-of-view, DA fouling leads, via the formation of a cascade of intermediates, to the polymerization of a melanin-like insulating substrate $[13,16]$. This polymer is mechanically strong, and similar to the glue secreted by mussels, which allows them to stick to virtually any organic or inorganic materials [17]. The adhesion of this substance is partly based on the properties of the amino acid 3,4-dihydroxy-L-phenylalanine (DOPA). This molecule can form covalent bonds to organic surfaces, and strong non-covalent bonds to inorganic surfaces [17]. For instance, DOPA was found to strongly adhere to $\mathrm{TiO}_{2}$ surfaces. Hence, it is possible that the melanin-like film polymerized onto the BDD surface makes stronger bonds with some organic moieties present at the surface of the anodically-treated surface. Hydrogen bonds have been found to play a role in DOPA adhesion to $\mathrm{TiO}_{2}$, and may therefore also contribute to fouling [18]. On the other hand, adhesion to the cathodically-prepared surface may occur via hydrophobic interactions [19].

\section{Conclusions}

Altogether, the data presented here suggest that cathodic preparation may offer the best performances for the electroanalysis of neurotransmitters. This pretreatment leads to enhanced electrochemical performances, as well as an improved stability during long DA oxidation, in conditions where more traditional carbon materials (such as glassy carbon or carbon fibres) would have been dramatically passivated. Nevertheless, the fact that the present study was carried out on the same sample, prepared in different manners, emphasizes the role of surface interaction in resistance to fouling, and that 
the stability of BDD in biological conditions may largely arise from its surface termination, rather than its diamond nature.

This system can be potentially be used as a model for explaining the mechanisms of bio-adhesion.

\section{Acknowledgements}

Funding of this work was provided by the EPFL and the EU Ideas program (ERC-2012-AdG-320404).

\section{References}

[1] R. Trouillon, D. O'Hare, Y. Einaga, Phys. Chem. Chem. Phys. 13 (2011) 5422.

[2] A.N. Patel, P.R. Unwin, J.V. Macpherson, Phys. Chem. Chem. Phys. 15 (2013) 18085.

[3] R. Trouillon, D. O'Hare, Electrochimica Acta 55 (2010) 6586.

[4] K. Yoshimi, Y. Naya, N. Mitani, T. Kato, M. Inoue, S. Natori, T. Takahashi, A. Weitemier, N. Nishikawa, T. McHugh, Y. Einaga, S. Kitazawa, Neurosci. Res. 71 (2011) 49.

[5] A. Suzuki, T.A. Ivandini, K. Yoshimi, A. Fujishima, G. Oyama, T. Nakazato, N. Hattori, S. Kitazawa, Y. Einaga, Anal. Chem. 79 (2007) 8608.

[6] B.A. Patel, Analyst 133 (2008) 516.

[7] H. Zhao, X. Bian, J.J. Galligan, G.M. Swain, Diam. Relat. Mater. 19 (2010) 182.

[8] C.-C. Chang, L.-C. Chen, S.-J. Liu, H.-C. Chang, J. Phys. Chem. B 110 (2006) 19426.

[9] H.B. Suffredini, V.A. Pedrosa, L. Codognoto, S.A.S. Machado, R.C. Rocha-Filho, L.A. Avaca, Electrochimica Acta 49 (2004) 4021.

[10] W. Miao, Z. Ding, A.J. Bard, J. Phys. Chem. B 106 (2002) 1392

[11] L.A. Hutton, J.G. Iacobini, E. Bitziou, R.B. Channon, M.E. Newton, J.V. Macpherson, Anal. Chem. 85 (2013) 7230.

[12] M.C. Granger, G.M. Swain, J. Electrochem. Soc. 146 (1999) 4551.

[13] W. Harreither, R. Trouillon, P. Poulin, W. Neri, A.G. Ewing, G. Safina, Anal. Chem. 85 (2013) 7447.

[14] A.J. Bard, R.L. Faulkner, Electrochemical Methods: Fundamentals and Applications, 2nd ed., John Wiley and Sons, Hoboken, NJ, USA, 2001.

[15] M.C. Rossi, F. Spaziani, S. Salvatori, G. Conte, Phys. Status Solidi A 199 (2003) 71.

[16] H. Lee, S.M. Dellatore, W.M. Miller, P.B. Messersmith, Science 318 (2007) 426.

[17] H. Lee, N.F. Scherer, P.B. Messersmith, Proc. Natl. Acad. Sci. 103 (2006) 12999.

[18] S.-C. Li, L.-N. Chu, X.-Q. Gong, U. Diebold, Science 328 (2010) 882.

[19] J. Yu, Y. Kan, M. Rapp, E. Danner, W. Wei, S. Das, D.R. Miller, Y. Chen, J.H. Waite, J.N. Israelachvili, Proc. Natl. Acad. Sci. U. S. A. 110 (2013) 15680. 


\section{Figure Captions}

Figure 1: A) XPS spectra obtained for the (top) cathodic and (bottom) anodic preparations. The O1s and C1s peaks are indicated. Typical CVs performed in B) $1 \mathrm{mM} \mathrm{FCMeOH}$ and C) $1 \mathrm{mM}$ DA (in aerated PBS, scan rate $100 \mathrm{mV} \mathrm{s}^{-1}$ ) for the cathodically ( $-3 \vee$, solid line) and anodically ( $+3 \vee$, dashed line) pretreated surfaces. D) Typical fouling curves observed in $1 \mathrm{mM}$ DA in aerated PBS for the cathodically ( $-3 \mathrm{~V}$, solid line) and anodically $(+3 \mathrm{~V}$, dashed line) pretreated surfaces. The insert shows a focus of the flat section of the curves emphasizing the slightly higher current observed for the cathodicallytreated electrode.

Figure 2: Typical CVs performed in A, B) $1 \mathrm{mM} \mathrm{FCMeOH}$ and C, D) $1 \mathrm{mM} \mathrm{DA}$ (in aerated PBS, scan rate $100 \mathrm{mV} \mathrm{s}^{-1}$ ) for the $A, C)$ cathodically $(-3 \mathrm{~V})$ and $B, D)$ anodically $(+3 \mathrm{~V})$ pretreated surfaces. The graphs show CVs performed before (solid line) and after (dashed line) a 15-minute fouling procedure in $1 \mathrm{mM}$ DA in aerated PBS. Typical EIS scans performed in $1 \mathrm{mM}$ DA in aerated PBS for E) cathodically $(-3 \mathrm{~V})$ and F) anodically $(+3 \mathrm{~V})$ pretreated surfaces. The graphs show traces obtained before (solid line) and after (dashed line) a 15-minute fouling procedure in $1 \mathrm{mM}$ DA in aerated PBS. 
Table

Table 1: CV characteristics (peak current $i_{p}$ and peak separation $\Delta E_{p}$ ) obtained in $1 \mathrm{mM}$ RuHex, $1 \mathrm{mM}$ FcMeOH and $1 \mathrm{mM}$ DA (in aerated PBS, scan rate $100 \mathrm{mV} \mathrm{s}^{-1}$ ) and EIS data (solution resistance $R_{\mathrm{s}}$ and charge-transfer resistance $R_{c t}$ ) obtained in $1 \mathrm{mM} \mathrm{DA}$, before and after a 15-minute fouling procedure in $1 \mathrm{mM}$ DA, where applicable. The BDD surfaces were cathodically $(-3 \mathrm{~V})$ and anodically $(+3 \mathrm{~V})$ pretreated. The charge transferred during the pretreatment $\left(Q_{p}\right)$ is also reported. The characteristics could not be determined (nd) for the CVs after fouling on the anodically treated surface, as an oxidation peak could not be observed anymore on the $\mathrm{CV}$. The values presented here are averages \pm standard deviation, for 3 independent measurements. The datasets were compared using double-tailed Student's t-test. The level for significance was $p<0.01$ and is indicated by \#\# (cathodic vs. anodic treatments before fouling) and ** (before vs. after fouling). A non-significant variation is indicated by -.

\begin{tabular}{|c|c|c|c|c|c|c|c|c|c|c|}
\hline & \multirow[t]{2}{*}{$Q_{p} / C$} & \multicolumn{2}{|c|}{$1 \mathrm{mM}$ RuHex } & \multirow[t]{2}{*}{ Fouling } & \multicolumn{2}{|c|}{$1 \mathrm{mM}$ FcMeOH } & \multicolumn{4}{|c|}{$1 \mathrm{mM} \mathrm{DA}$} \\
\hline & & $i_{p} / \mu \mathrm{A}$ & $\Delta E_{p} / \mathrm{mV}$ & & $i_{p} / \mu \mathrm{A}$ & $\Delta E_{p} / \mathrm{mV}$ & $i_{p} / \mu \mathrm{A}$ & $\Delta E_{p} / \mathrm{mV}$ & $R_{S} / \Omega$ & $R_{c t} / \mathrm{k} \Omega$ \\
\hline \multirow[t]{2}{*}{$-3 V$} & \multirow[t]{2}{*}{$-14 \pm 3$} & \multirow[t]{2}{*}{$26.1 \pm 1.9$} & \multirow[t]{2}{*}{$78 \pm 3$} & Before & $21.6 \pm 0.7$ & $73 \pm 3$ & $61.1 \pm 1.8$ & $322 \pm 8$ & $350 \pm 30$ & $6.2 \pm 0.6$ \\
\hline & & & & After & $12.4 \pm 1.1^{\star *}$ & $118 \pm 3^{\star *}$ & $26 \pm 11^{\star *}$ & $750 \pm 110^{* *}$ & $530 \pm 50^{\star *}$ & $35 \pm 10^{* *}$ \\
\hline \multirow[t]{2}{*}{$+3 \mathrm{~V}$} & \multirow[t]{2}{*}{$1.3 \pm 0.1^{\# \#}$} & \multirow[t]{2}{*}{$26.0 \pm 0.8^{-}$} & \multirow[t]{2}{*}{$83 \pm 3^{-}$} & Before & $17 \pm 3^{-}$ & $100 \pm 20^{-}$ & $39.2 \pm 1.5^{\# \#}$ & $530 \pm 70^{\# \#}$ & $510 \pm 30^{\# \#}$ & $11.0 \pm 1.2^{\# \#}$ \\
\hline & & & & After & $4.4 \pm 0.5^{\star \star}$ & $230 \pm 30^{* *}$ & nd & nd & $1180 \pm 50^{* *}$ & $75 \pm 9^{\star \star}$ \\
\hline
\end{tabular}


A) XPS

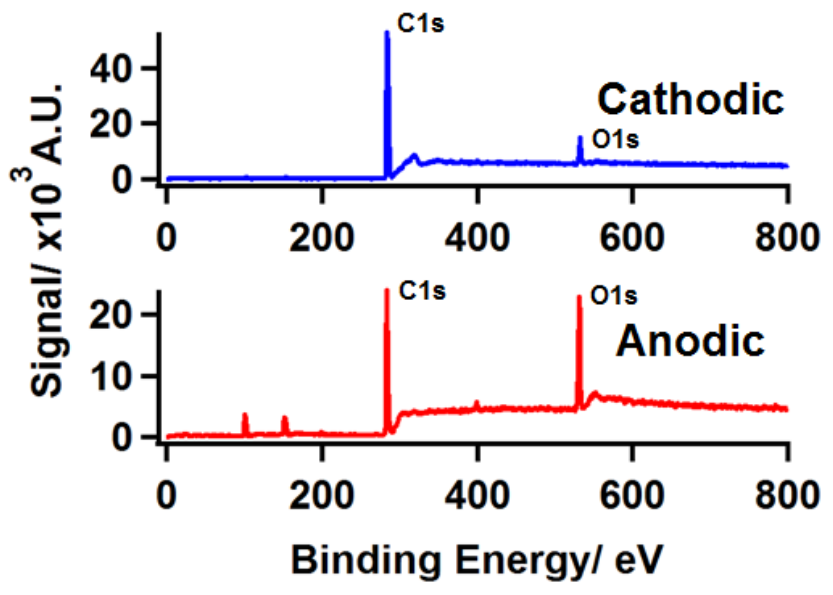

C) $1 \mathrm{mM}$ dopamine

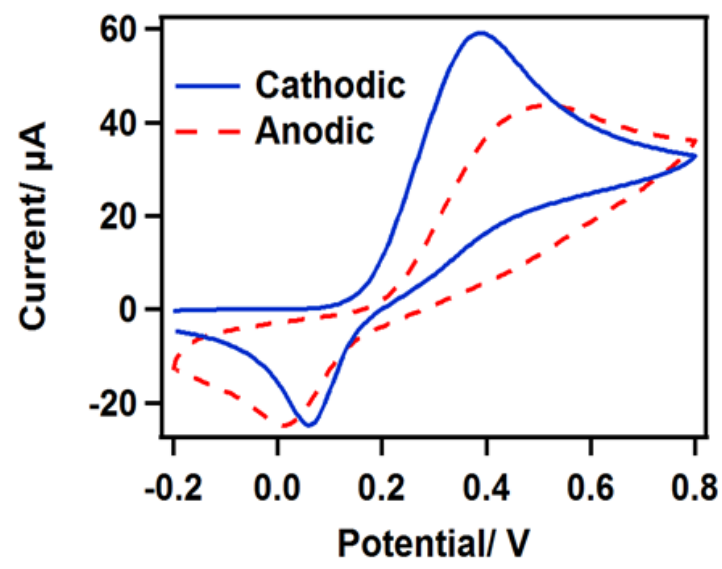

\section{B) $1 \mathrm{mM} \mathrm{FcMeOH}$}

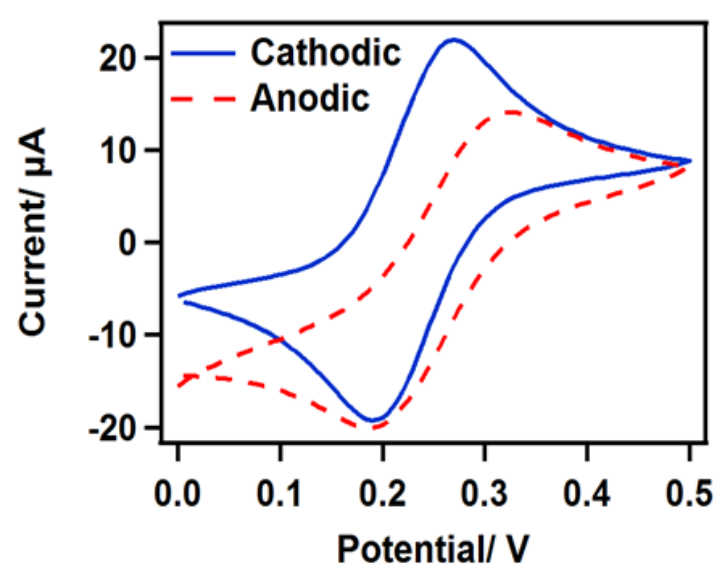

D) Fouling tests

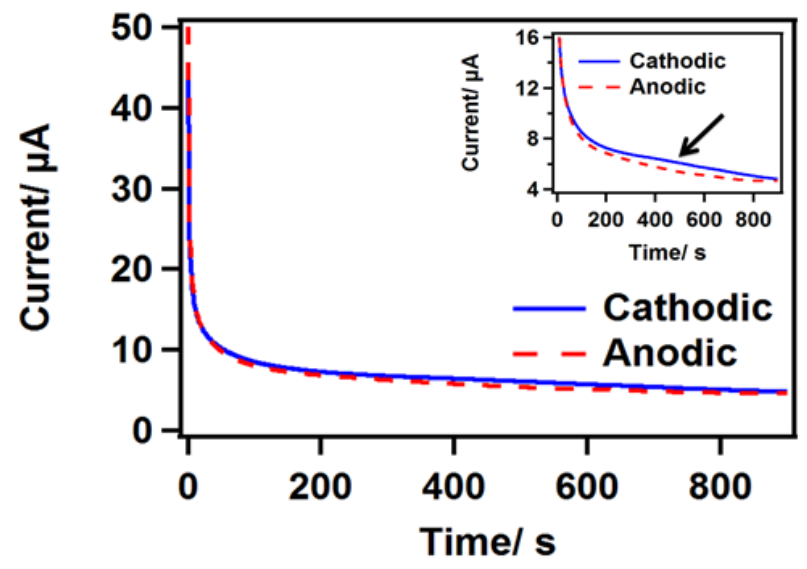


Cathodic (-3 V)
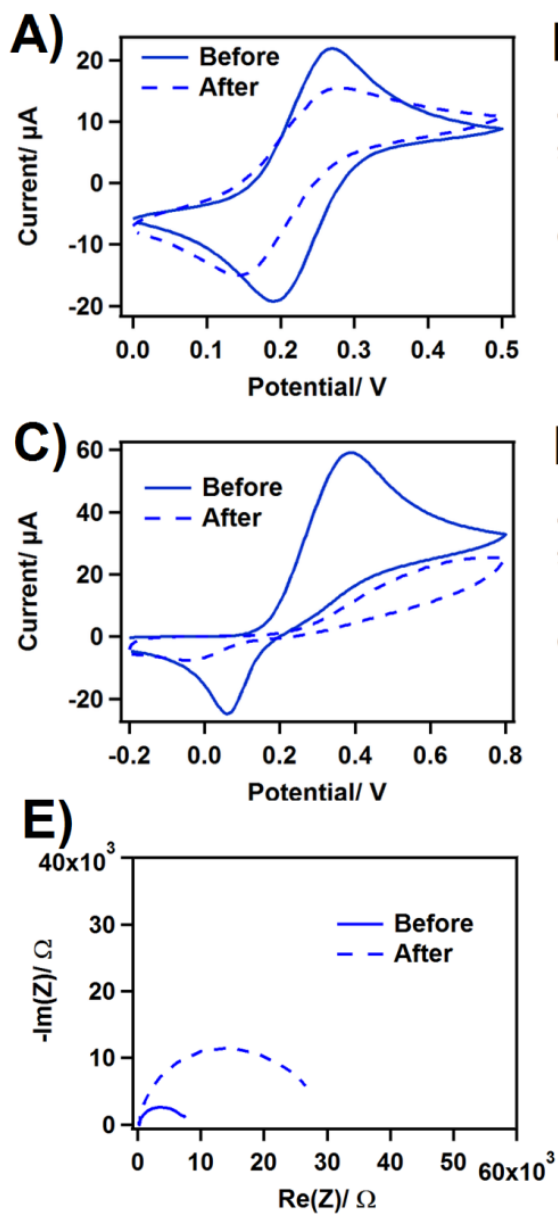

Anodic (+3 V)
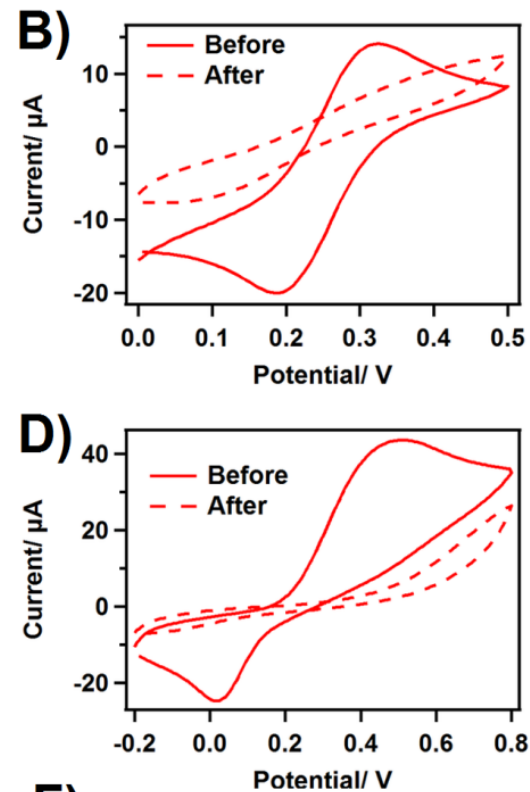

F)

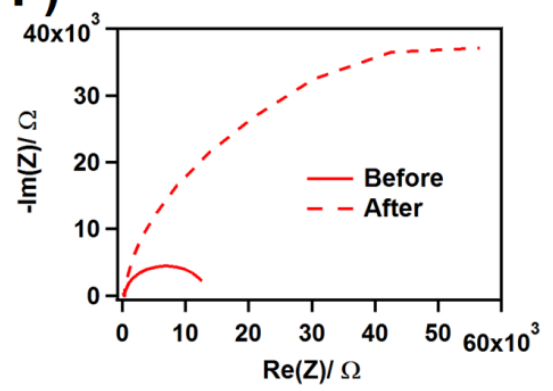

